\title{
Effect of Geometry of Filler Particles on the Effective Thermal Conductivity of Two-Phase Systems
}

\author{
Deepti Chauhan", Nilima Singhvi, Ramvir Singh \\ Department of Physics, Heat Transfer Laboratory, University of Rajasthan, Jaipur, India \\ Email: *chouhan.deepti275@gmail.com
}

Received March 24, 2012; revised April 22, 2012; accepted April 30, 2012

\begin{abstract}
The present paper deals with the effect of geometry of filler particles on the effective thermal conductivity for polymer composites. In the earlier models, less emphasis has been given on the shape of filler particles. In this paper, expressions for effective thermal conductivity has been derived using the law of minimal thermal resistance and equal law of the specific equivalent thermal conductivity for three different shapes i.e. spherical, elliptical and hexagonal of filler particles respectively. Calculated values of effective thermal conductivity for various samples using the derived expressions then compared with experimental data available and other models developed in the literature. The results calculated are in good agreement with the earlier experimental data and the deviation, is least in our expressions showing the success of the model.
\end{abstract}

Keywords: Effective Thermal Conductivity; Polymer Composites; Minimal Thermal Resistance; Shape of Filler

\section{Introduction}

Particle filled polymer composites have become important because of their wide applications in science and engineering for technological developments. Incorporating inorganic fillers into a matrix enhances various physical properties of the materials such as mechanical strength, elastic modulus and heat transfer coefficient $[1,2]$. In general, the mechanical properties of particulate filled polymer composites depend strongly on size, shape and distribution of filler particles in the polymer matrix [3].

Composite materials have primarily been used for structural applications. Polymer composite materials have been found extremely useful for heat dissipation applications like in electronic packaging, in computer chips [1, 2]. Polymer composites filled with metal particles are of interest for many fields of engineering. The interest in these composite materials arises from the fact that the thermal characterization of such composites are close to the properties of metals, whereas the mechanical properties and the processing methods are typical for plastics [3, 4]. Adding fillers to plastics, changes the behaviour of polymers and a significant increase in the effective thermal conductivity of the system has been observed $[5,6]$.

Therefore, it is very important to understand the heat transfer mechanism in polymer composites. Maxwell [7] calculated the effective thermal conductivity of a random distribution of spheres in a continuous medium, which

"Corresponding author. worked well for low filler concentrations. Bruggeman [8] derived another model for the effective thermal conductivity, under different assumptions for permeability and field strength. Hamilton and Crosser [9] extended Maxwell's model to include the empirical factor $\mathrm{n}$ to account for the shape of the particles $(n=3$ for spheres and $n=6$ for the cylinders). Liang and Liu [10] gave a theoretical model for evaluating the effective thermal conductivity of inorganic particulate polymer composites. Liang and Lia [11] measured the effective thermal conductivity $\left(K_{\text {eff }}\right)$ of hollow glass-bead (HGB) filled polypropylene composites by means of a thermal conductivity instrument to identify the effect of the content and size of the $\mathrm{HGB}_{\mathrm{s}}$ on the effective thermal conductivity $\left(K_{\text {eff }}\right)$. Tekce et al. [12] studied the thermal conductivity of copper filled polyamide composites using the Hot-Disk method in the range of filler content $0 \%-30 \%$ by volume for short fibers and $50 \%-60 \%$ by volume for particle shape of plates and spheres. Nielson [13], Cheng and Vachon [14] and Agari et al. [15] proposed various theoretical models for describing the heat transfer mechanism in polymer composites. Liang and Lia [16] studied the heat transfer mechanism in inorganic hollow micro-spheres filled polymer composites and proposed a heat transfer model.

In the present paper, expressions for effective thermal conductivity $\left(K_{\text {eff }}\right)$ of various inorganic particles filled polymer composites has been developed for different shapes of filler particles as spherical, elliptical and hex- 
agonal using the law of minimal thermal resistance and equal law of the specific equivalent thermal conductivity. The calculations were done for samples and compared with the experimental results available in the literature.

\section{Theory}

There are several mathematical models for predicting the effective thermal conductivity of particle filled polymer composites like Maxwell model, Russell model, Hamilton and Crosser model and others [7-17]. Some of them are as under:

Maxwell model

$$
K_{\text {eff }}=K_{m} \frac{K_{f}+2 K_{m}+2 \phi\left(K_{f}-K_{m}\right)}{K_{f}+2 K_{m}-\phi\left(K_{f}-K_{m}\right)}
$$

Russell model

$$
K_{e f f}=K_{m}\left[\frac{\phi_{f}^{\frac{2}{3}}+\frac{K_{m}}{K_{f}}\left(1-\phi_{f}^{\frac{2}{3}}\right)}{\phi_{f}^{\frac{2}{3}}-\phi_{f}+\frac{K_{m}}{K_{f}}\left(1-\phi_{f}^{\frac{2}{3}}\right)}\right]
$$

Hamilton and Crosser model

$$
K_{\text {eff }}=\frac{K_{f}+(n-1) K_{m}+(n-1)\left(K_{f}-K_{m}\right) \phi}{K_{f}+(n-1) K_{m}-\left(K_{f}-K_{m}\right) \phi} K_{m}
$$

Here $K_{m}$ and $K_{f}$ are the conductivity coefficients of the polymer matrix and the filler particles, and $n$ is an empirical factor to account for the shape of the filler particles, $(n=3)$ for spheres and $(n=6)$ for cylinders. These models are basically developed for dilute dispersion.

Liang and Liu [10] established a theoretical model of inorganic particulate-filled polymer composites. This model is based on the specific equivalent thermal resistance of the element of composites, when only heat conduction is considered. Therefore, the calculation of the equivalent thermal conductivity for composites can be attributed to the determination of the equivalent thermal conductivity of the unit cell with the same specific equivalent thermal resistance.

In this paper, we start with the assumption that an overall composite consists of a number of small squared unit cells having sides $\mathrm{H}$ and each cell contains only one filler particle which can be of different shapes as spherical, elliptical and hexagonal, kept at the center of the unit cell. We also assumed that the heat flow into the element is from the top of the square. A series model of heat conduction through the unit cell in an inorganic particulate filled polymer composite is shown in Figure 1.

To study the effect of various shapes of filler particles on the effective thermal conductivity $\left(K_{e f f}\right)$ of the sys-

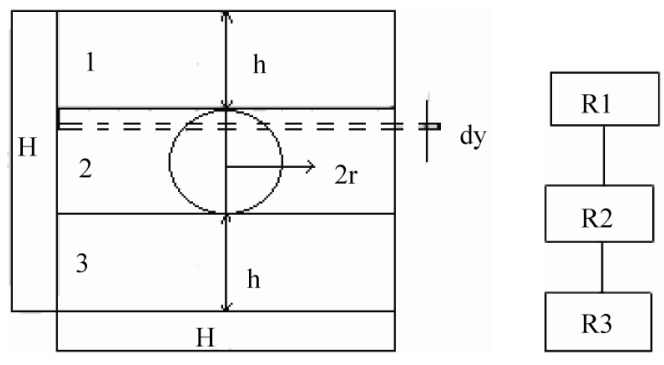

(a)
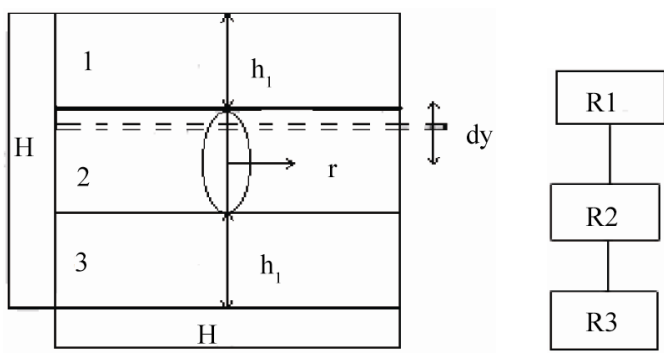

(b)
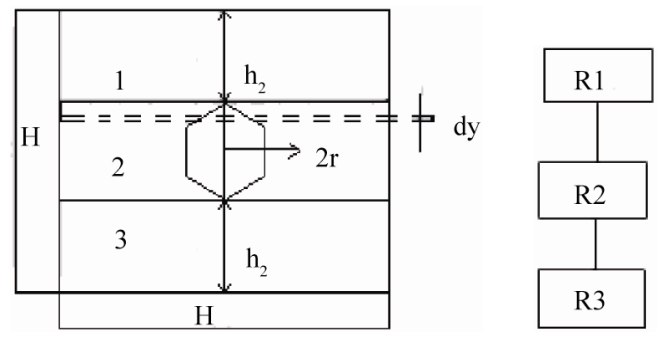

(c)

Figure 1. Heat transfer series model for different shapes of filler particles.

tem, we assumed that the filler particle is located at the center of the unit cell. The cell is then divided into three parts as shown in Figure 1. The mean thermal conductivity of the matrix and the filler are $K_{p}$ and $K_{f}$ respectively. The quantity of heat flowing through a body depends upon the heat transfer route in the materials. The equivalent thermal resistance of these three parts for different shapes is given as:

For spherical shape

$$
\begin{aligned}
& R_{1}=R_{3}=\frac{h}{K_{p} A} \\
& R_{2}=\frac{4 r^{2}}{\left(K_{p} V_{p}+K_{f} V_{f}\right)}
\end{aligned}
$$

and

Here

and

$$
V_{p}=2 H^{2} r-1.33 \pi r^{3}
$$

For elliptical shape 


$$
R_{1}=R_{3}=\frac{h_{1}}{K_{p} A}
$$

and

$$
R_{2}=\frac{r^{2}}{\left(K_{p} V_{p}+K_{f} V_{f}\right)}
$$

Here

$$
V_{p}=2.828 H^{2} r-0.166 \pi r^{3}
$$

and

$$
V_{f}=0.166 \pi r^{3}
$$

For hexagonal shape

$$
\begin{aligned}
& R_{1}=R_{3}=\frac{h_{2}}{K_{p} A} \\
& R_{2}=\frac{4 r^{2}}{\left(K_{p} V_{p}+K_{f} V_{f}\right)}
\end{aligned}
$$

and

Here

and

$$
\begin{aligned}
& V_{p}=2 H^{2} r-2.43 \sqrt{3} r^{3} \\
& V_{f}=2.43 \sqrt{3} r^{3}
\end{aligned}
$$

Here $A$ is the total cross-sectional area of the unit cell, $V_{p}$ and $V_{f}$ are the volume of polymer matrix and filler particles, and $r$ is a variable parameter defined in Figures 1(a)-(c) respectively. by

The total equivalent thermal resistance is then given

$$
R=R_{1}+R_{2}+R_{3}
$$

Solving Equations (4)-(15) and keeping the volume of the unit cell for different shapes same as that of the spherical shape, the expressions for effective thermal conductivity are obtained for spherical, elliptical and hexagonal shapes as under:

For spherical shape of filler particles, the equation is

$$
K_{\text {eff }}=\frac{1}{\frac{1}{K_{p}}-\frac{1}{K_{p}}\left(\frac{6 \phi_{f}}{\pi}\right)^{\frac{1}{3}}+\frac{2}{K_{p}\left(\frac{4 \pi}{3 \phi_{f}}\right)^{\frac{1}{3}}+\left(\frac{2 \phi_{f}}{9 \pi}\right)^{\frac{1}{3}} \pi\left(K_{f}-K_{p}\right)}}
$$

For elliptical shape of filler particles, the equation is

$$
K_{e f f}=\frac{1}{\frac{1}{K_{p}}-\frac{1}{K_{p}}\left(\frac{6 \phi_{f}}{\pi}\right)^{\frac{1}{3}}+\frac{2}{\frac{K_{p}}{\sqrt{2}}\left(\frac{\pi}{6 \phi_{f}}\right)^{\frac{1}{3}}+\left(\frac{2 \phi_{f}}{9 \pi}\right)^{\frac{1}{3}} \pi\left(K_{f}-K_{p}\right)}}
$$

and for hexagonal shape of filler particles, the equation is

$$
K_{\text {eff }}=\frac{1}{\frac{1}{K_{p}}-\frac{1.23}{K_{p}}\left(\phi_{f}\right)^{\frac{1}{3}}+\frac{2}{1.62 \frac{K_{p}}{\left(\phi_{f}\right)^{\frac{1}{3}}}+1.29\left(\phi_{f}\right)^{\frac{1}{3}}\left(K_{f}-K_{p}\right)}}
$$

Here $\phi_{f}$ is the volume fraction of filler in the resin matrix.

\section{Results and Discussion}

In this paper, we have obtained semi empirical relations for effective thermal conductivities given by Equations (17)-(19). These equations have been derived taking into account the different shapes of filler particles as spherical, elliptical and hexagonal. And then the calculations were done considering these shapes. The calculated values are then compared with the experimental data available in the literature for phenol-aldehyde/graphite, phenol-aldehyde/aluminum oxide, polypropylene/aluminum and poly- propylene/copper composites with volume fraction ranging from 0 - 0.5. Figures 2-5 show the comparison between the predictions by Equations (1)-(3) and the experimental data of the effective thermal conductivity of the phenol-aldehyde/graphite, phenol-aldehyde/aluminum oxide, polypropylene/aluminum and polypropylene/copper composites. The values of the thermal conductivities of the samples used are given in Table 1.

The graphs represented in Figures 2-5 show that value of $K_{\text {eff }}$ calculated by the Equations (17)-(19) were closer to the experimental data then calculated with other models given by Equations (1)-(3). Most of the models fail to predict the effective thermal conductivity over the entire range of filler concentration. It is seen that Maxwell 


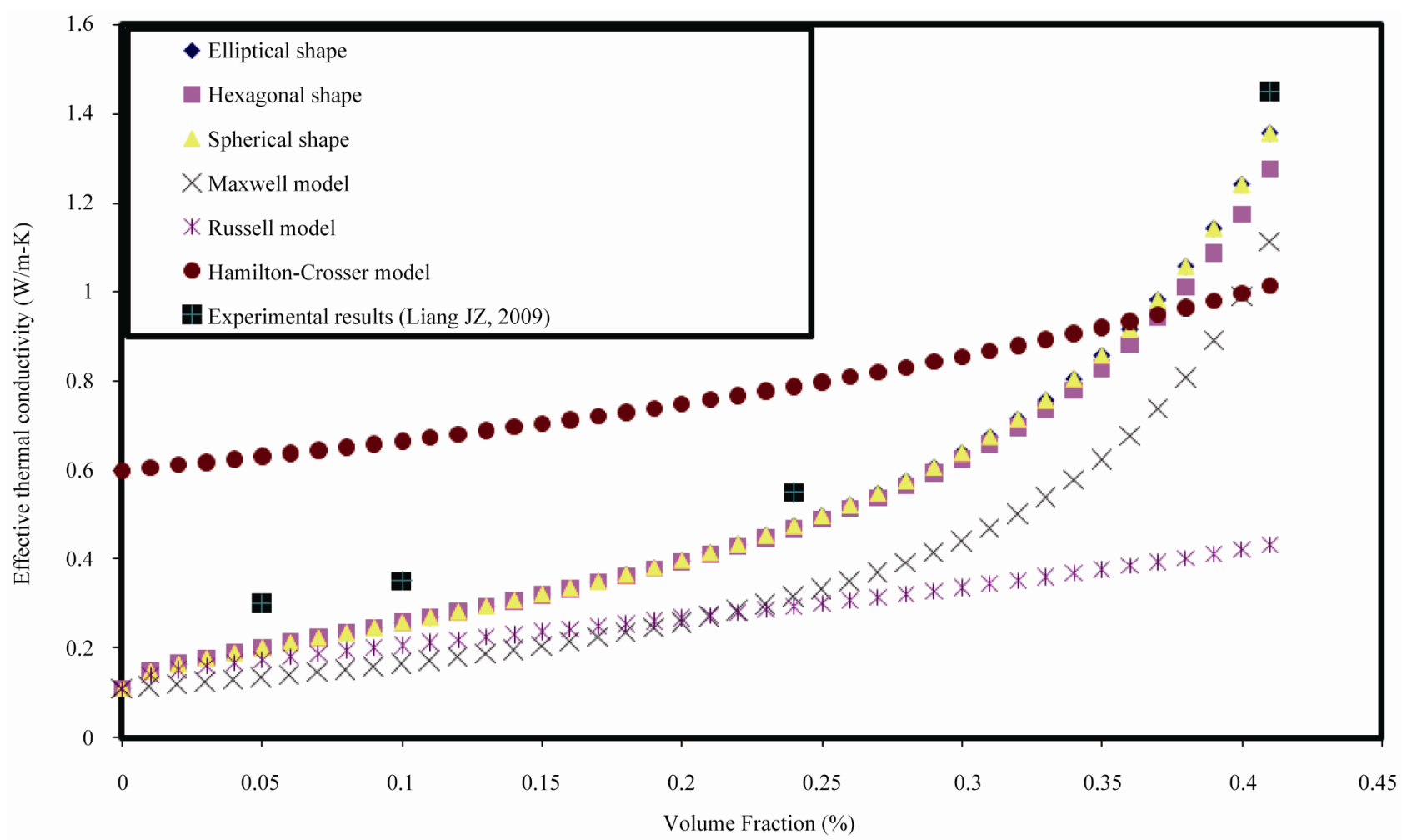

Figure 2. Variation of effective thermal conductivity and filler volume fraction of phenol-aldehyde/graphite composites for different shapes.

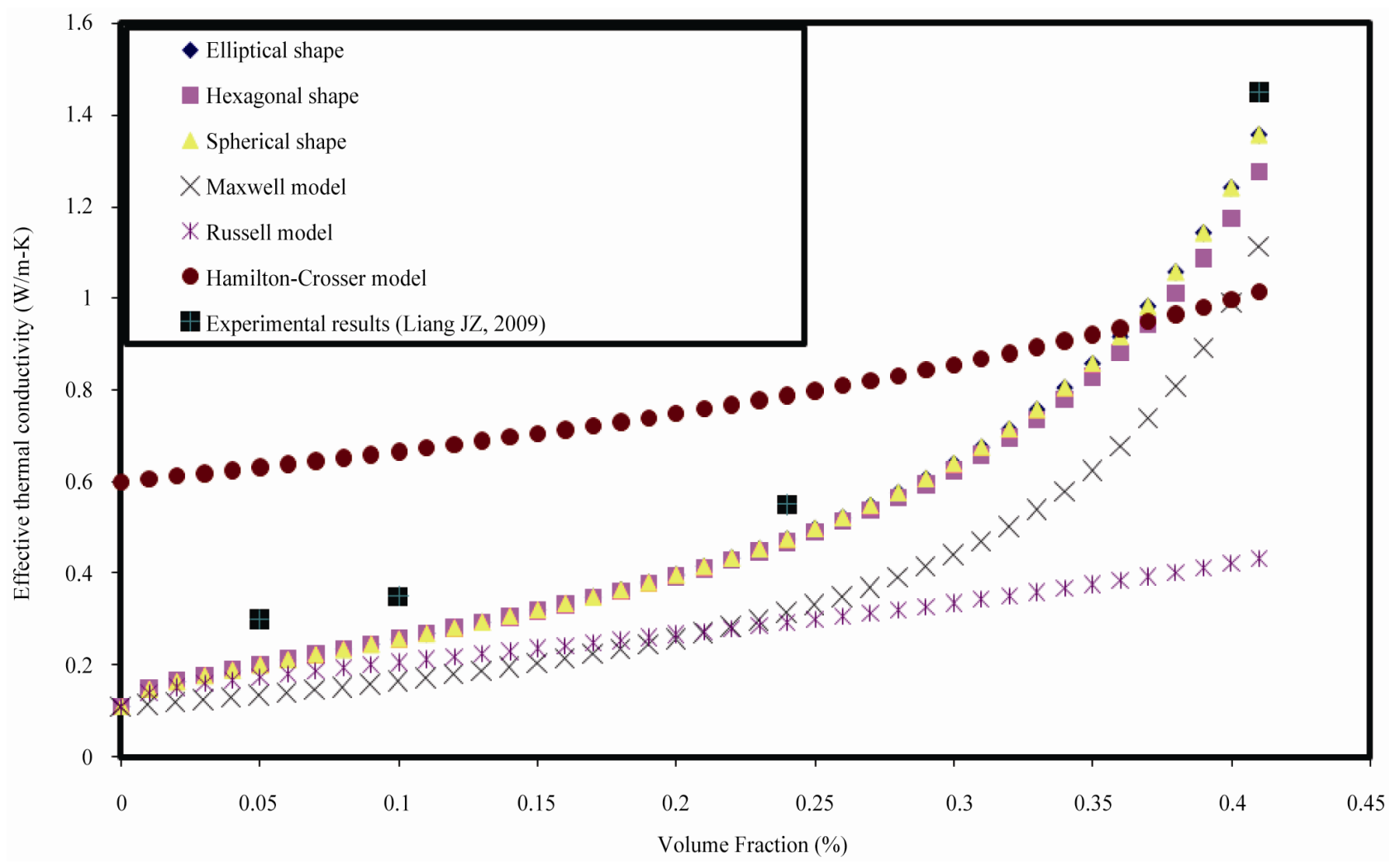

Figure 3. Variation of effective thermal conductivity and filler volume fraction of phenolaldehyde/aluminum oxide composites for different shapes. 


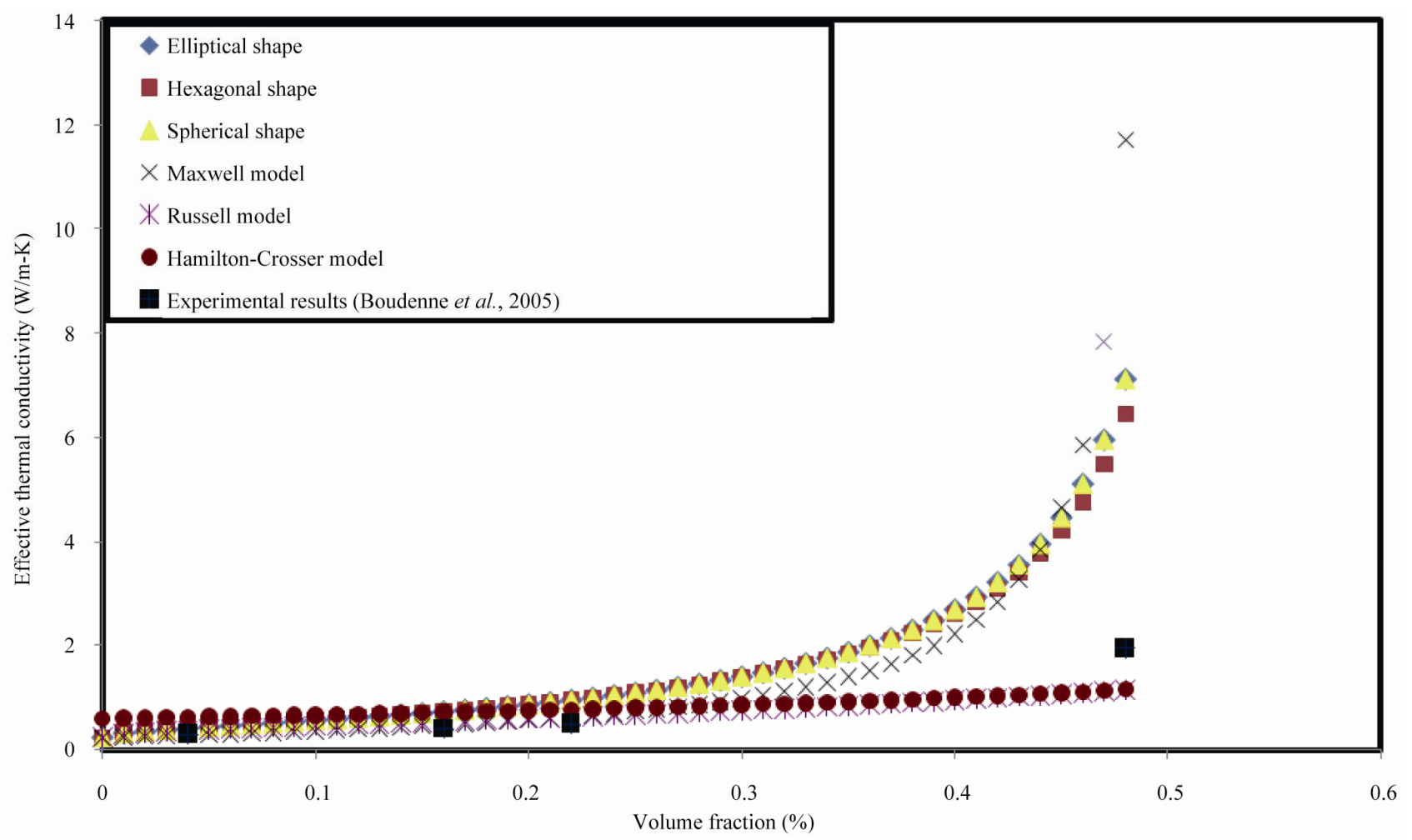

Figure 4. Variation of effective thermal conductivity and filler volume fraction of polypropylene/aluminum composites for different shapes.

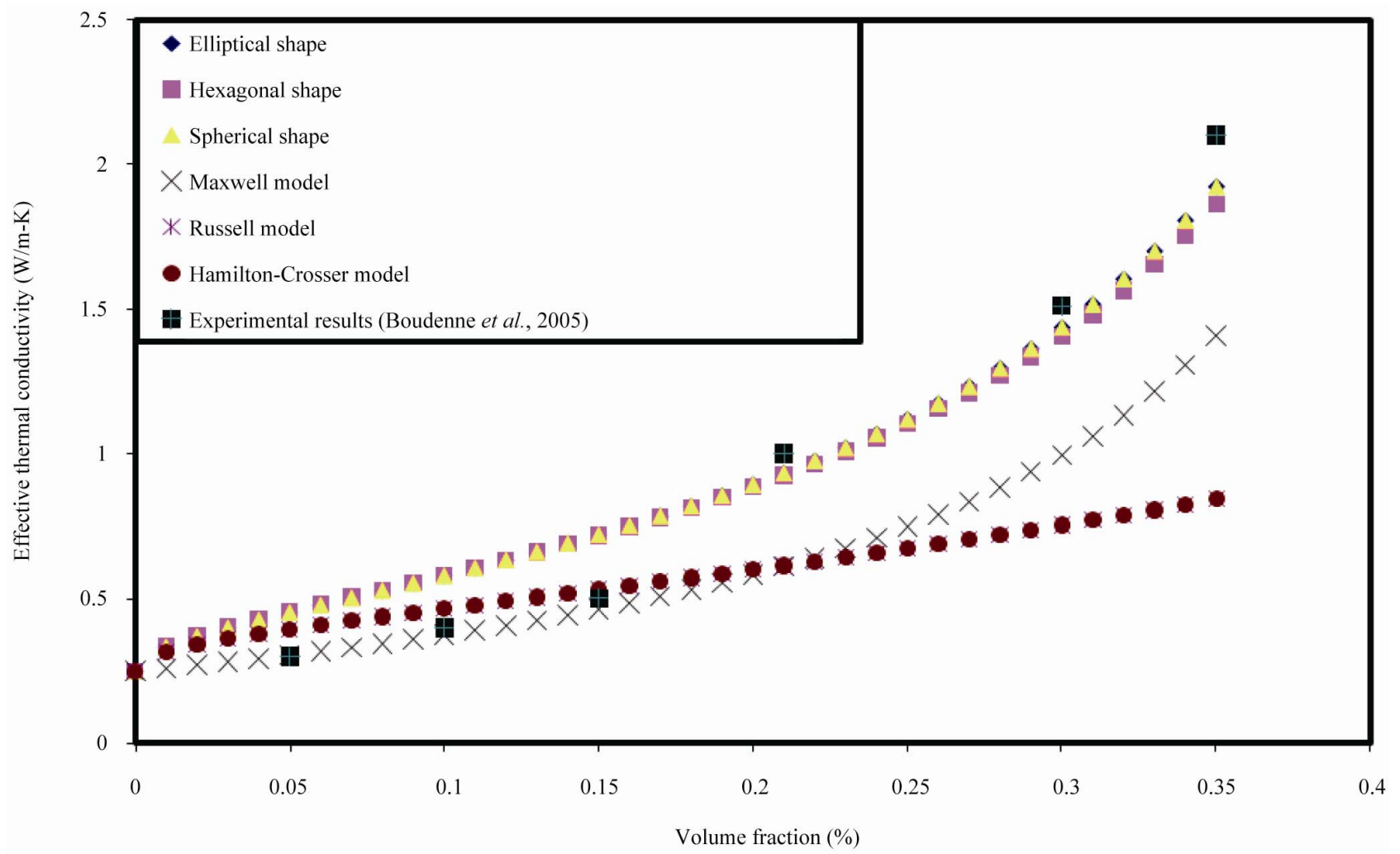

Figure 5. Variation of effective thermal conductivity and filler volume fraction of polypropylene/copper composites for different shapes. 
Table 1. Thermal conductivity of various samples used in our computations.

\begin{tabular}{cccc}
\hline Polymer Matrix & Thermal Conductivity $(\mathrm{W} / \mathrm{m}-\mathrm{K})$ & Filler & Thermal Conductivity $(\mathrm{W} / \mathrm{m}-\mathrm{K})$ \\
\hline Phenol Aldehyde & 0.111 & Graphite & 120 \\
Phenol Aldehyde & 0.111 & Aluminium Oxide & 204 \\
Polypropylene & 0.25 & Aluminium & 237 \\
Polypropylene & 0.25 & Copper & 387 \\
\hline
\end{tabular}

and Russell equations under estimate the experimental data over the entire range of filler concentrations, while the Hamilton and Crosser model (with $n=3$, spherical shape of fillers) over estimates the effective thermal conductivity of the materials for phenol-aldehyde/graphite and phenol aldehyde/aluminum oxide composites. It can also be seen that, the calculations done by our model for different shapes are closer to the experimental data then done by any other theoretical model predicted earlier Equations (1)-(3). It is seen from the Figures 2-5 that with the increase in the filler concentration, the effective thermal conductivity of the composite increases. The effective thermal conductivity values of the samples used for our computations are mentioned in Table 1. Table 1 show that the thermal conductivity of the fillers is significantly higher than that of the matrix. There is a significant change in the $K_{\text {eff }}$ of the composite systems as we change the shape of the filler particles. It is seen from the figures that the $K_{\text {eff }}$ increases nonlinearly with an increase in the volume fraction of filler particles.

Sphericity is a measure of the roundness of the particle, denoted by $\psi$. The value of $\psi$ is one for spherical particles and less than one for other shapes of the particles. The change in the geometrical configuration of the particles changes the value of sphericity. An increase in the value of $\psi$ means that there is decrease in the angularity, which brings the particles closer and there is an increase in the $K_{\text {eff }}$ of the system. This change in the sphericity can be seen in the form of small deviations among the theoretical curves drawn for different shapes as spherical, elliptical and hexagonal Figures 2-3.

Hence, it is observed that the theoretical values for $K_{\text {eff }}$ calculated by our model proposed for different shapes, with other existing mathematical models Equations (1)-(3) are in good agreement with each other for low volume fractions i.e. $\phi<0.3$. This is because of the low dispersion of particles in the matrix, due to which the particles are not able to interact with each other. However, when the volume fraction increases more than 0.3 , there is a rapid increase in the $K_{\text {eff }}$ of the system as the particles come closer as such the interaction between filler particles become stronger.

\section{Conclusions}

Theoretical expressions for effective thermal conductivity for the polymer composites which were established, was based on the law of minimal thermal resistance and the equal law of the specific equivalent thermal conductivity. The different shapes of filler particles as spherical, elliptical and hexagonal were considered for the calculations. Equations (17)-(19) gives the relationship between the effective thermal conductivity ( $K_{\text {eff }}$ ) of filled polymer composites in terms of inclusion volume fraction as well as other physical parameters of polymer matrix and fillers when filler volume fraction is less than $60 \%$.

The values of the effective thermal conductivity $\left(K_{\text {eff }}\right)$ of phenol-aldehyde/graphite, phenol aldehyde/aluminum oxide, polypropylene/aluminum and polypropylene/copper composites were then calculated. Good agreement was found between the theoretical estimations and experimental data available in the literature. We further plan to verify these formulae for some more samples.

\section{REFERENCES}

[1] C. P. Wong and R. S. Bollampally, "Thermal Conductivity, Elastic Modulus, and Coefficient of Thermal Expansion of Polymer Composites Filled with Ceramic Particles for Electronic Packaging," Journal of Applied Polymer Science, Vol. 74, No. 14, pp. 3396-3403.

doi:10.1002/(SICI)1097-4628(19991227)74:14<3396::AI D-APP13>3.0.CO;2-3

[2] X. Lu and G. Xu, "Thermally Conductive Polymer Composites for Electronic Packaging," Journal of Applied Polymer Science, Vol. 65, No. 13, 1997, pp. 2733-2738. doi:10.1002/(SICI)1097-4628(19970926)65:13<2733::AI D-APP15>3.0.CO;2-Y

[3] H. Tavman, "Thermal and Mechanical Properties of Copper Powder Filled Poly (Ethylene) Composites," Powder Technology, Vol. 91, No. 1, 1997, pp. 63-67. doi:10.1016/S0032-5910(96)03247-0

[4] A. Boudenne, L. Ibos, E. Gehin, M. Fois and J. C. Majeste, "Anomalous Behavior of Thermal Conductivity and Diffusivity in Polymeric Materials Filled with Metallic Particles," Journal of Material Science, Vol. 40, No. 16, 2005, pp. 4163-4167. doi:10.1007/s10853-005-3818-2

[5] N. M. Sofian, M. Rusu, R. Neagu and E. Neagu, "Metal Powder-Filled Polyethylene Composites. V. Thermal Properties," Journal of Thermoplastic Composite Materials, Vol. 14, No. 1, 2001, pp. 20-33. doi:10.1106/9N6K-VKH1-MHYX-FBC4

[6] B. Weidenfeller, M. Hofer and F. R. Schilling, "Thermal Conductivity, Thermal Diffusivity, and Specific Heat Capacity of Particle Filled Polypropylene," Composites Part 
A: Applied Science and Manufacturing, Vol. 35, No. 4, 2004, pp. 423-429.

doi:10.1016/i.compositesa.2003.11.005

[7] J. C. Maxwell, "A Treatise on Electricity and Magnetism," 3rd Edition, Dover, New York, 1954.

[8] G. Bruggeman, "Calculation of Various Physics Constants in Heterogeneous Substances I Dielectricity Constants and Conductivity of Mixed Bodies from Isotropic Substances," Annalen der Physik, Vol. 416, No. 7, 1935, pp. 636-664. doi:10.1002/andp.19354160705

[9] R. L. Hamilton and O. K. Crosser, "Thermal Conductivity of Heterogeneous Two-Component Systems," Industrials and Engineering Chemistry Fundamentals, Vol. 1, No. 3, 1962, pp. 187-191. doi:10.1021/i160003a005

[10] J. Z. Liang and G. S. Liu, "A New Heat Transfer Model of Inorganic Particulate-Filled Polymercomposites," Journal of Material Science, Vol. 44, No. 17, 2009, pp. 47154720. doi:10.1007/s10853-009-3729-8

[11] J. Z. Liang and F. H. Li, "Measurement of Thermal Conductivity of Hollow Glass-Bead-Filled Polypropylene Composites," Polymer Testing, Vol. 25, No. 4, 2006, pp. 527531. doi:10.1016/j.polymertesting.2006.02.007

[12] H. S. Tekce, D. Kumlutas and I. H. Tavman, "Effect of Particle Shape on Thermal Conductivity of Copper Reinforced Polymer Composites," Journal of Reinforced Plas- tics and Composites, Vol. 26, No. 1, 2007, pp. 113-121. doi: $10.1177 / 0731684407072522$

[13] L. E. Nielson, "Thermal Conductivity of Particulate-Filled Polymers," Journal of Applied Polymer Science, Vol. 17, No. 12, 1973, pp. 3819-3820. doi:10.1002/app.1973.070171224

[14] S. Cheng and R. Vachon, "A Technique for Predicting the Thermal Conductivity of Suspensions, Emulsions and Porous Materials," International Journal of Heat Mass Transfer, Vol. 13, No. 3, 1970, pp. 537-546. doi:10.1016/0017-9310(70)90149-3

[15] Y. Agari, A. Ueda and S. Nagai, "Thermal Conductivity of a Polymer Composite," Journal of Applied Polymer Science, Vol. 49, No. 9, 1993, pp. 1625-1634. doi:10.1002/app.1993.070490914

[16] J. Z. Liang and F. H. Li, "Simulation of Heat Transfer in Hollow Glass- Bead-Filled Polypropylene Composites by Finite Element Method," Polymer Testing, Vol. 26, No. 3, 2007, pp. 419-424.

doi:10.1016/j.polymertesting.2006.12.014

[17] T. B. Lewis and L. E. Nielson, "Dynamic Mechanical Properties of Particulate-Filled Composites," Journal of Applied Polymer Science, Vol. 14, No. 6, 1970, pp. 14491471. doi:10.1002/app.1970.070140604 